ESJ Humanities

\title{
Les labyrinthes borgésiens : Nature et caractéristiques
}

\author{
Majida Sayegh
}

Faculté des Lettres et des Sciences Humaines (Section 5)

Département de Langue et Littérature Françaises, Université Libanaise

Doi:10.19044/esj.2022.v18n7p36

Submitted: 15 December 2021

Accepted: 17 February 2022

Published: 28 February 2022
Copyright 2022 Author(s)

Under Creative Commons BY-NC-ND

4.0 OPEN ACCESS

Cite As:

Sayegh M. (2022). Les labyrinthes borgésiens : Nature et caractéristiques. European Scientific Journal, ESJ, 18 (7), 36. https://doi.org/10.19044/esj.2022.v18n7p36

\section{Résumé}

Le présent article tente d'identifier la notion du labyrinthe selon une méthode synthétique et exploratrice du texte littéraire. En effet, les caractéristiques d'un labyrinthe borgésien ne se limitent pas à l'aspect matériel ou architectural, mais il couvre les différentes variables qui affectent la vie humaine : l'errance dans l'ignorance, la peur de l'oubli, la stagnation de la pensée, l'emprisonnement dans la particularité, les conflits avec soi-même et avec l'autre, l'absence d'une interaction fructueuse entre l'homme et le savoir et la lutte pour dominer autrui. Pratiquement, déambuler dans un labyrinthe moral ou physique, spatial ou temporel, engendre des cheminements de solitude, d'angoisse incessante, de perturbation due aux diverses sources d'enchevêtrement inextricable, de désordre générateur de confusion et d'extrême complication. Malgré les souffrances endurées par les victimes prises au piège, le labyrinthe peut se révéler, parfois, comme leur itinéraire initiatique pour atteindre la libération suprême et la vérité radicale.

Mots-clés : Labyrinthe, physique, moral, temporel, spatial, infini, bifurcation, libération 


\title{
Borgesian labyrinths: Definition and characteristics
}

\author{
Majida Sayegh \\ Faculty of Letters and Human Sciences (Branch 5) \\ Department of French Language and Literature, Lebanese University
}

\begin{abstract}
The present article attempts to identify the notion of the labyrinth according to a synthetic and exploratory method of the literary text. Indeed, the characteristics of a Borgesian labyrinth are not limited to the material or architectural aspects as it covers the different factors affecting human life. These aspects are the wandering in ignorance, the fear of forgetting, the stagnation of thought, the imprisonment in specifics, the conflicts within one's self and with others, the absence of a fruitful interaction between man and knowledge, and the struggle to dominate others. Wandering in a moral, physical, spatial or temporal labyrinth, generates paths of solitude and of incessant anguish. It also leads to disruption due to the various sources of inextricable entanglement, confusion and extreme complication. Despite the suffering endured by the trapped victims, the labyrinth can sometimes prove to be an initiation towards supreme liberation and the radical truth.
\end{abstract}

Keywords: Labyrinth, physical, moral, temporal, spatial, infinite, divergence, liberation

\section{Introduction}

Jorge Luis Borges (1899-1986), amoureux de complétude cosmique et passionné de littérature fantastique, se caractérise par un style énigmatique et symbolique cherchant à explorer l'état de l'homme encerclé par toutes sortes de labyrinthes fruits de son ignorance devant la complexité du monde. Martin (2006) dans son livre, Borges. Une biographie de l'éternité, parle d'un homme qui « voulait décrire la vie de tous les hommes, de tous les temps et de tous les lieux en mélangeant histoire et fantastique ». Il a commencé son travail comme bibliothécaire à Buenos Aires, puis il est devenu le directeur de la Bibliothèque nationale. Grâce à une vision profonde et illimitée, il a créé son propre monde littéraire. La plupart de ses nouvelles sont courtes et gorgées de mystères évoquant le conflit de la finitude humaine avec l'immensité du cosmos.

Lorsque le thème de labyrinthe et sa signification est abordé, le chercheur est invité à considérer toutes les expériences humaines comme champ d'investigation. En effet, malgré les découvertes scientifiques et leur vulgarisation, les lois qui orchestrent l'univers restent une énigme pour l'homme. C'est pourquoi, le labyrinthe ne peut pas se réduire à la signification 
uniquement matérielle. En effet, l'entendement des hommes erre toujours dans un monde privé de coordonnées ou contenant des composantes trompeuses. Effectivement, la pensée humaine continue de tourner dans le grand labyrinthe de la vérité racine et chaque fois qu'elle progresse sur son chemin vers l'origine dont l'identité est ignorée, elle découvre l'ampleur du chemin qui reste à parcourir.

L'être humain est paradoxalement le constructeur et la victime de son propre labyrinthe dont il essaie de se délivrer grâce à ses réflexions et son imagination. C'est ce que Kremer-Marietti (1999) essaie de dire quand elle écrit : « Le labyrinthe renferme le chemin de l'origine, celui qui conduit à la racine dernière, la vérité radicale [...]. Mais le labyrinthe a été construit par les hommes pour y cacher cette réalité ». L'homme exerce sa faculté pensive afin de sortir de ce labyrinthe de la pensée par la pensée elle-même qui est en soi un vrai labyrinthe, par conséquent il reste égaré dans les couloirs du savoir à la recherche d'une issue finale. Ceci évoque le fil d'Ariane confié à Thésée pour l'affranchir de son labyrinthe. Mais jamais l'homme « ne revient de son labyrinthe. Seul Thésée en est revenu pour se perdre dans Ariane, son nouveau

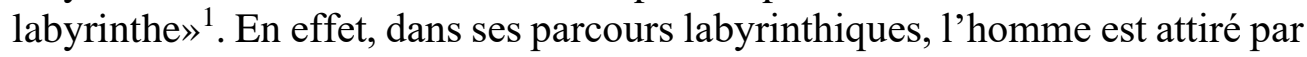
l'espoir d'atteindre la vérité racine. Pourtant, malgré ses douloureux efforts, il n'arrive qu'à la périphérie lointaine de cette vérité. C'est pourquoi, et dès le début, l'homme doit se préparer intellectuellement et moralement à affronter le chaos comme préconise Sager (2014).

En dehors de sa dimension cosmique, la vision labyrinthique de Borges est certainement corrélée avec sa biographie et ses expériences réelles ou imaginaires. À cet égard, Martin (2006) écrit : «Lire la vie de Borges, c'est parcourir avec lui les labyrinthes d'un monde dont, tel un "dieu bibliothécaire", il a dessiné les contours, déployé le désert, engendré par jets d'encre les populations, construites les villes, où chaque individu est comme l'hologramme d'une pensée, né des visions d'un homme aveugle.»

Le labyrinthe borgésien possède plusieurs dimensions : matérielle, spirituelle, symbolique et temporelle. En effet, la façon de le concevoir consiste à en masquer la sortie en adoptant le principe des répétitions à l'identique. Face à cette similitude, la victime n'a plus de repères et son cheminement devient chaotique ce qui aboutit à l'errance. Même dans les cauchemars, le rêveur vit comme s'il était entré dans un labyrinthe en exprimant son inquiétude par des cris de détresse et des mouvements incontrôlés.

Le thème du labyrinthe matérialisé ou non est omniprésent dans la plupart des œuvres de Borges. Avant la traduction française de l'œuvre

${ }^{1}$ Kremer-Marietti, A. (1970). L'homme labyrinthique et la vérité. Les Études Philosophiques(1), 47-61. 
L'Aleph de Borges, Roger Caillois avait traduit et publié quatre récits du recueil Fictions (1944) dans un livre intitulé Labyrinthes (1955) composé de quatre nouvelles: L'Immortel, Histoire du Guerrier et de la Captive, L'Écriture du Dieu et La Quête d'Averroës. Quelle est la vision borgésienne du labyrinthe ? Ce dernier possède-t-il des dimensions qui sont propres à Borges ? Le concept du labyrinthe touche-t-il seulement sa construction matérielle ou concerne-t-il essentiellement l'esprit de l'homme et sa recherche de la vérité radicale ? Chez Borges, la pensée humaine est-elle un labyrinthe ? Si oui comment Borges justifie-t-il son raisonnement ?

Selon une méthode synthétique basée sur le contenu du texte luimême, cet article vise à explorer les images labyrinthiques, explicites ou implicites, surgies de la pensée borgésienne dans de nombreuses nouvelles, en accordant une attention particulière à La Demeure d'Astérion qui offrent une originalité de la conception du labyrinthe.

\section{Les images labyrinthiques surgies de la pensée borgésienne}

La notion du labyrinthe a évolué avec le temps. Dans le dictionnaire Larousse $^{2}$, un labyrinthe est un " réseau compliqué de chemins, de galeries dont on a du mal à trouver l'issue». Au sens figuré ${ }^{3}$, un labyrinthe peut désigner un ensemble embrouillé et confus. Le labyrinthe évoque principalement un parcours semé d'embûches. Selon Maryse Marsailly ${ }^{4}$, «son symbolisme rejoint celui de l'entrelacs, dont les points de croisement des tracés renvoient par ailleurs à l'idée de tissage ». Le labyrinthe était assimilé au madrépore par Buttor (1975) et à un « réseau qui participe du rhizome» par Deleuze et Gattarie (1980). C'est un «édifice composé d'un grand nombre de chambres et de passages disposés tellement, qu'une fois engagé, on n'en pouvait trouver l'issue » d'après Taillade (1994).

Pourtant, les labyrinthes borgésiens dépassent les caractéristiques classiques de ces définitions. Selon Sieber (2004), « le labyrinthe de Borges est le symbole d'un système dans lequel il y a deux objectifs : sortir du labyrinthe et s'immerger complètement dans le labyrinthe ». Dans l'Immortel $l^{5}$, Borges écrit qu'un «labyrinthe est une chose faite à dessein pour confondre les hommes ; son architecture, prodigue en symétries, est orientée à cette intention (23) ». Il ajoute qu' « il n'est pas de plaisir plus complexe que celui de la pensée et c'est à celui-là que nous nous consacrions (31)». Cela conduit à considérer que la pensée humaine révèle un labyrinthe d'extrême complexité, et ceci concorde avec Nicolas (1999) lorsqu'il affirme que « le premier labyrinthe de Borges, c'est sa pensée ».

\footnotetext{
${ }^{2}$ https://www.larousse.fr/dictionnaires/francais/labyrinthe/45804\#.

${ }^{3} \mathrm{https} / / /$ fr.thefreedictionary.com/dédales.

${ }^{4} \mathrm{https} / / /$ blogostelle.blog/category/histoire-du-sacre/

${ }^{5}$ Borges, L'immortel (Fictions, Gallimard, 1957, pour la traduction française).
} 
En effet, la toile de l'araignée reste un symbole vivant de l'architecture labyrinthique. Cet animal arthropode détient le secret de déplacement sur son filet qui n'est qu'un piège affreux pour ses victimes. Borges utilise le réseau de l'araignée comme un indice du labyrinthe. Ceci est repéré dans sa nouvelle Abenhacan el Bokhari mort dans son labyrinthe ${ }^{6}$ où il écrit : «El Bokhari rêva dans un tombeau d'un réseau de serpents et qu'il découvrit à son réveil qu'une toile d'araignée lui avait suggéré ce songe (166)». Dans l'Aleph ${ }^{7}$ aussi :

Chaque chose équivalait à une infinité de choses, parce que je la voyais clairement de tous les points de l'univers. Je vis la mer populeuse, l'aube et le soir, les foules d'Amériques, une toile d'araignée argentée au centre d'une noire pyramide, un labyrinthe brisé (c'était Londres) [...] des tigres, des pistons, des bisons, des foules des armées [...] je vis toutes les fourmis qu'il y a sur la terre [...] je vis l'Aleph, sous tous les angles, je vis sur l'Aleph la terre, et sur la terre de nouveau l'Aleph et sur l'Aleph la terre (208-209).

Ainsi, Borges emploie le comptage des séries infinies des choses pour évoquer le labyrinthe de la pensée humaine. Si la répétition d'un mot dans un poème « ravive l'idée » comme le dit Augustin Pélissier ${ }^{8}$, chez Borges, le "grand poème" est l'univers. Effectivement, la répétition de vingt-quatre fois « je vis » évoque l'infini. L'auteur voit la répétition de chaque chose suivant des séries illimitées qui peuvent se représenter de différentes manières et dans des conditions spatio-temporelles très variées : la mer, la terre ferme, la succession de la nuit et du jour, la toile d'araignée, le labyrinthe de Londres, les troupeaux d'animaux rassemblés les uns avec les autres, le labyrinthe de l'illusion, les caravanes de fourmis. Le narrateur tente de nous faire savoir qu'il y a des séries infinies de créatures et de choses qui se communiquent à travers des langages libérés de symboles qui s'unifient en un seul langage cosmique.

L'Aleph et la terre sont inséparables. Ils parlent un langage qui n'a pas besoin d'alphabet, dépassant les limites de chaque objet, et que l'esprit humain perçoit lorsqu'il se dissout dans "l'esprit de l'univers". Dans cette optique, Duborgel (1982) écrit : " L'Aleph nie les lois de la langue, de la logique et de l'espace. Abolition de l'ordre spatio-temporel, des vecteurs haut-bas, gauche

\footnotetext{
${ }^{6}$ Borges, Abenhacan el Bokhari mort dans son labyrinthe (l'Aleph, Gallimard, 1967, pour la traduction française)

${ }^{7}$ Borges, L'Aleph (L'Aleph, op. cit.)

${ }^{8}$ Pélissier Augustin, Principes de rhétorique française, http://obvil.parissorbonne.fr/corpus/ecole/pellissier_principes-de-rhetoriquefrancaise_1867/body4?q=r\%C3\%A9p\%C3\%A9tition\#mark1, 1873, np.
} 
droite, devant-derrière, avant-après échappe au langage humain ». Ainsi, toutes les langues humaines, anciennes et modernes, et qui ne sont pas encore nées, ne sont qu'un murmure confus de cette incroyable langue de l'univers dont l'alphabet est infini. Véritablement, dans l'œuvre borgésienne, le lecteur peut repérer « des Langues et des Signes étranges. Souvent, ses héros sont en quête d'une Parole ou d'un Mot unique qui aurait la puissance de dire ou de contenir l'univers. Rêve d'un Signe-Microcosme, miroir du monde » comme affirme Mourey (2013).

Le rêve libérateur de Borges consisterait à découvrir la formule idéale qui contient tous les secrets et tous les pouvoirs de l'univers à l'instar de la formule divine de quatorze mots de L'Écriture $d u D^{2 i e u}{ }^{9}$ qui a permis à Tzinancan de sortir de tous ses labyrinthes pour s'unifier à l'univers tout entier. En fait, le thème du temps accompagne souvent celui du labyrinthe ce qui conduit à une chaine de labyrinthes qui s'engendrent sans cesse sous la puissante autorité du hasard. Dans son avertissement du recueil L'Aleph, Roger Caillois écrit :

Ces couloirs qui bifurquent et qui ne mènent à rien qu'à des salles identiques aux premières et d'où rayonnent ces couloirs homologues, ces répétitions oiseuses, ces duplications épuisantes enferment l'auteur dans un labyrinthe qu'il identifie volontiers avec l'univers. Où que l'homme se tienne, lui semble$t-i l$, il se trouve toujours au centre d'indiscernables reflets, d'inextricables correspondances ; à perte de vue, de conscience, ce sont gemmations et scissiparités, harmoniques et allitérations : premiers termes de séries impérieuses et vaines, absurdes, désespérantes, annulaires peut-être (p.8, 9).

Dans L'Immortel ${ }^{10}$, Borges décrit le labyrinthe qui conduit à la Cité des immortels, la «Cité surhumaine (20) », comme « un chaos de galeries sordides [...]. Cette cave avait neuf portes ; huit introduisaient à un labyrinthe qui, insidieusement, ramenait à la même chambre. La neuvième (grâce à un autre labyrinthe) donnait sur une seconde chambre circulaire, identique à la première (21) ». Pour décrire un univers labyrinthique, l'auteur évoque la bifurcation comme avec ces « cryptes à neuf portes » et ces « longs souterrains qui se ramifient (22)». De plus, l'architecture de cette Cité « était antérieure aux hommes, antérieur à la Terre. Cette ostensible antiquité $[\ldots]$ me parut convenable à l'ouvrage d'artisans immortels (23) ». Ses descriptions «présentent le dispositif classique du réseau inextricable » comme mentionne

\footnotetext{
${ }^{9}$ Borges, L'Aleph, Gallimard, 1967, pour la traduction française.

${ }^{10}$ Borges, L'immortel (L'Aleph, Gallimard, 1967, pour la traduction française).
} 
Taillade (1994). Le monde des immortels serait le prototype du labyrinthe car « la similitude s'imposait partout de telle sorte qu'une pièce représentait toutes les autres pièces, un couloir ne se distinguait pas des autres couloirs, ce qui mettait la raison en faillite totale et la paralysait $»^{11}$.

Le principe de l'analogie, comme constituant du dédale, s'approfondit avec Les Deux Rois et les Deux Labyrinthes ${ }^{12}$ qui illustre un conflit étrange entre le roi de Babylonie et le roi des Arabes qui disposent de deux visions opposées concernant la confusion et l'émerveillement liés à la conception du labyrinthe. Le roi de Babylonie « réunit ses architectes et ses mages et leur ordonna de construire un labyrinthe si complexe et si subtil que les hommes les plus sages ne s'aventureraient pas à y entrer et que ceux qui y entreraient s'y perdraient (171) ». Son intention était d'égarer le roi des Arabes pour se montrer supérieur et plus puissant, et probablement pour se débarrasser de lui. Après s'être sauvé miraculeusement du labyrinthe babylonien, ce dernier jure de rendre un jour le coup à son adversaire car il possédait en Arabie un labyrinthe naturel encore plus déroutant. La lutte entre les deux rois symbolise la concurrence entre deux types de labyrinthes : le premier a été construit avec perfection grâce aux meilleurs architectes de l'époque et avec la participation des mages, tandis que le deuxième était tout simplement le désert sans repères, un labyrinthe façonné par la nature, au-dessus du niveau du sol et en plein air. En effet, ce piège naturel cause la perte du constructeur du labyrinthe artificiel qui révèle ses limites devant l'étendue de la nature. Dans cette courte nouvelle, l'auteur met l'accent sur l'intérêt du labyrinthe naturel qui le fascine.

Cet émerveillement borgésien s'exprime à maintes reprises surtout avec dans $L^{\prime} E_{\text {criture }} d u$ Dieu $^{13}$ qui se distingue par le foisonnement de types labyrinthiques dans lesquels vit son protagoniste, le prêtre Tzinacan, emprisonné dans un trou effroyable. Anéanti et effacé dans son corps vieillissant et martyrisé, il est réduit à un pur esprit qui essaie de s'affranchir de toutes les conditions réductrices par tous les moyens : la mémoire, le rêve et même les taches sur la peau d'un jaguar qui partage l'autre moitié de son cachot circulaire. Plusieurs types de labyrinthes matériels et abstraits apparaissent dans cette nouvelle où «le labyrinthe devient un constituant naturel de la manifestation de la vie cosmique ${ }^{14}$ : le trouble de la mémoire du vieux prisonnier Tzinacan, sa torture répétitive par ses bourreaux, son incapacité de déchiffrer "l'énigme concrète" symbolisée par les taches noires

\footnotetext{
${ }^{11}$ Sayegh M. (2021). L'Immortel de Borges ou le refus de l'éternité. European Scientific Journal, ESJ, 17(6), 130-149.

${ }^{12}$ Borges, Les Deux Rois et les Deux Labyrinthes (l'Aleph, Gallimard, 1967, pour la traduction française)

${ }^{13}$ Borges, L'Écriture du Dieu, (L'Aleph, op. cit.)

${ }^{14}$ Sayegh M. (2021). L'Écriture du Dieu : Libération de la pensée humaine des banalités telluriques. European Scientific Journal, ESJ, 17 (33), 43-65.
} 
sur le pelage jaune du jaguar, la recherche mystérieuse de la sentence divine composée de quatorze mots et les cauchemars des grains de sable et leur chaine interminablement emboitée. Ces différents labyrinthes reflètent la variété de la nature labyrinthique à laquelle Tzinacan échappe grâce à la roue de l'eau et du feu qui dévoile tous les secrets.

Il est à noter que le labyrinthe final dans lequel s'enfonce Tzinacan est libérateur et va à l'encontre de tous les autres qui le torturaient. L'univers, par son immensité est un labyrinthe bienfaisant car il affranchit l'être humain de ses dimensions insignifiantes pour devenir une partie intégrante de l'abîme cosmique. En fait, l'image labyrinthique de l'univers est idéalisée par Borges puisqu'elle représente à ses yeux l'infinitude absolue, parfaite et inimitable. Cette idée fait écho à celle abordée dans la nouvelle Les Deux Rois et les Deux labyrinthes mettant en valeur le labyrinthe naturel opposé au labyrinthe artificiel. Effectivement, incernable matériellement et théoriquement, le macrocosme s'harmonise bien avec le concept du labyrinthe borgésien. Dans ce contexte, dans la nouvelle Abenhacan el Bokhari mort dans son labyrinthe $e^{15}$, l'auteur écrit: « Il n'est pas nécessaire de construire un labyrinthe quand l'Univers déjà en est un (165).»

L'idée de la perfection est corollaire du dédale chez Borges qui emprunte aux mathématiques des conceptions fines et évocatrices: le labyrinthe construit par Abenhacan est apparu « comme une paroi rectiligne et presque interminable [...] [et] qu'elle était de forme circulaire, mais que le rayon en était si vaste que la courbure devenait imperceptible (156)». L'usage des notions mathématiques est très fréquent dans le raisonnement déductif de Borges dont la performance est remarquable quand il écrit que «toute ligne droite est l'arc d'un cercle infini (157)». Mais mathématiquement parlant, la droite a une seule dimension, et le cercle a deux dimensions, donc les deux sont parfaitement définis et repérés dans l'espace. En fait, et comme l'estime Nicolas (1999), le cercle rappelle « la quête sans fin, la limite asymptotique vers une droite $»$.

En fait, « le cercle et l'infini sont l'âme du labyrinthe» estime Mourey ${ }^{16}$ (2013) et les deux concepts ont leur place d'honneur dans l'imaginaire borgésien. La sphère est la forme la plus parfaite dans le monde réel de trois dimensions. Elle symbolise le mouvement selon Lavarde (1999) et elle est omniprésente dans les objets infiniment petits (ou infinitésimaux) et les objets infiniment grands. L'image fascinante de l'univers est en lien avec cette symbolique des notions mathématiques. En effet, le concept de l'infini est l'essence du labyrinthe chez Borges, c'est pourquoi la phrase liminaire de

\footnotetext{
${ }^{15}$ Borges, Abenhacan el Bokhari mort dans son labyrinthe (L'Aleph, Gallimard, 1967, pour la traduction française).

${ }^{16}$ https://agoras.typepad.fr/regard eloigne/jlborges/.
} 
La Bibliothèque de Babel ${ }^{17}$ est : "L'univers (que d'autres nomment la Bibliothèque) se compose d'un nombre indéfini, et peut-être infini, de galeries hexagonales, avec au centre de vastes puits d'aérations bordés par des balustrades très basses (79)». L'auteur ajoute : " La Bibliothèque est une sphère dont le centre véritable est un hexagone quelconque et dont la circonférence est inaccessible (81) ». Ainsi, Borges évoque implicitement un espace infini parce que le centre de cette sphère peut être n'importe quel hexagone, par conséquent la recherche d'un livre particulier ressemble à la recherche d'une issue d'un labyrinthe interminable. Cette conception de l'absolu n'est qu'un signe que l'homme est en face d'un savoir illimité objet d'une quête incessante. Dans ce sens va l'interrogation de Pascal (1954, p.84) : "Qu'est-ce que l'homme dans la nature ? Un néant à l'égard de l'infini, un tout à l'égard du néant, un milieu entre rien et tout, infiniment éloigné de comprendre les extrêmes ». L'expression "un milieu entre rien et tout" désigne le centre d'un cercle, source de l'égalité entre les hommes dans leurs actions éternelles.

Dans ses œuvres Histoire de l'éternité et Le temps circulaire ${ }^{18}$ Borges perçoit le temps comme un cercle où chaque point se confond avec lui-même après un tour complet dans une sorte de recommencement éternel qui oblige l'homme à revenir une infinité de fois par le même point. Il précise aussi que cette concordance entre les points de départ et d'arrivée transforme l'histoire universelle en une histoire d'un seul homme. En fait, le cercle évoque une diversité de symboles : "l'unité, l'harmonie, l'infini et le domaine de l'esprit. Il est à la fois début et fin, fini et infini, tout et rien ${ }^{19}$.»

Avec la nouvelle Le livre de sable ${ }^{20}$, le labyrinthe prend la nature d'un livre dont les pages sont innombrables et interchangeables. Cet objet insaisissable n'apporte aucune information cohérente au lecteur, c'est pourquoi il est décrit comme « un objet de cauchemar, une chose obscène qui diffamait et corrompait la réalité (133) ». En effet, chaque 2000 pages « une bifurcation surgit et par conséquent l'univers de ce livre est chaotique, or la force intellectuelle de l'homme est incapable de saisir l'attracteur de ce $\operatorname{livre}^{21} \ldots$.. Borges justifie le choix de ce titre en disant «...ni ce livre ni le sable n'ont de commencement ni de fin (130) ». Il ajoute «si l'espace est

\footnotetext{
${ }^{17}$ Borges, La Bibliothèque de Babel (Fictions, Gallimard, 1957, pour la traduction française) ${ }^{18}$ Borges, J.-L. (1936(1971)). Historia de la eternidad (Histoire de l'éternité), essai "El tiempo circular" (Le temps circulaire). trad. de l'espagnol par Roger Caillois et Laure Guille, Paris, U.G.E. Paris: UGE.

${ }^{19} \mathrm{https} / / / \mathrm{www} . j e p e n s e . o r g /$ symbolisme-du-cercle/

${ }^{20}$ Borges, Le livre de sable (Le livre de sable, 1978 pour la traduction française)

${ }^{21}$ Sayegh, M. (2021). Le Chaos borgésien entre réel et virtuel. European Scientific Journal, ESJ, 17 (36), 98-115.
} 
infini, nous sommes dans n'importe quel point de l'espace. Si le temps est infini, nous sommes dans n'importe quel point du temps (130)».

Cette idée est renforcée dans la nouvelle Le jardin aux sentiers qui bifurquent ${ }^{22}$ où le concept du labyrinthe semble lié au monde livresque aussi car le mystérieux Ts'ui Pên est l'auteur de ce fameux Jardin qui est « un vague amas de brouillons contradictoires » où le héros meurt au troisième chapitre et « au quatrième il est vivant (102) ». Tout le monde cherchait un mystérieux labyrinthe construit par cet auteur et «personne ne pensa que le livre et le labyrinthe étaient un seul objet (102)». Le narrateur, comme tout le monde, le cherchait et méditait :

Ce labyrinthe perdu, je l'imaginai inviolé et parfait au sommet secret d'une montagne, je l'imaginai effacé par des rizières ou sous l'eau; je l'imaginai infini, non plus composé de kiosques octogonaux et de sentiers qui reviennent, mais de fleuves, de provinces et de royaumes... Je pensai à un labyrinthe de labyrinthes, à un sinueux labyrinthe croissant qui embrasserait le passé et l'avenir et qui impliquerait les astres en quelque sorte (99).

Par ce passage, Borges évoque la nature multiple que peut revêtir un labyrinthe et met l'accent sur le facteur temporel. Dans cette nouvelle policière de Borges la relativité dans les conceptions et les mesures du temps crée une pépinière de labyrinthes qui renaissent à chaque ramification. En effet, la «bifurcation du jardin s'ouvre à la temporalité, et conduit à une pluralité de futurs [...] Autrement dit, un réseau de labyrinthes parallèles se forme et l'ensemble devient un labyrinthe de labyrinthes ${ }^{23}{ }$.

$\mathrm{Si}$ la confusion des dates et des identités peuvent être des caractéristiques du monde labyrinthique, la nouvelle Funes ou la mémoire ${ }^{24}$ prouve que le contraire peut l'être aussi. En fait, Funes qui « ne fréquentait personne » et «savait l'heure comme une montre (115)», dispose d'une mémoire surhumaine possédant une capacité illimitée. Il avait à lui seul «plus de souvenirs que n'en peuvent avoir eu tous les hommes depuis que le monde est monde (114) ». A l'aide de cette faculté sans trou, il est capable de restituer parfaitement les actions suivant leur chronologie avec leurs détails les plus minimes. Sa mémoire sans perturbation est visuelle aussi : par l'hyper-netteté elle est capable de vaincre l'errance engendrée de n'importe quel labyrinthe. Ainsi, ce pouvoir mémoriel presque robotique serait "l'anti-labyrinthe" par

\footnotetext{
${ }^{22}$ Borges, Le jardin aux sentiers qui bifurquent (Fictions, Gallimard, 1957, pour la traduction française).

${ }^{23}$ Sayegh, op. cit.

${ }^{24}$ Borges, Funes ou la mémoire (Fictions, op. ct.)
} 
excellence. Pourtant, restituer les événements à leur durée effective nécessite un temps équivalent de la part de celui qui raconte ainsi que de celui qui écoute, ce qui provoque la nausée et la confusion à ce dernier, malaises caractéristiques des dédales. C'est pourquoi, seule la mémoire sélective pourrait mettre terme à l'exhaustivité destructrice d'où les bienfaits de certain oubli pour l'homme car cette mémoire démesurée s'est transformée en « un tas d'ordure (114)». En effet, Funes a perdu son individualité pour devenir tous les hommes de tous les temps ce qui est à la fois monstrueux, fantastique et surtout insoutenable. Ce héros borgésien accablé par cette mémoire était incapable de penser car «penser c'est oublier des différences, c'est généraliser, abstraire. Dans le monde surchargé de Funes il n'y avait que des détails, presque immédiats (123) ». La mémoire de Funes serait l'histoire de l'humanité prise au piège des énumérations qui empêchent d'abstraire, c'està-dire de penser et de raisonner pour se métamorphoser en labyrinthe éternel.

Ainsi, les labyrinthes dépendent de multiples facteurs et ne se limitent jamais aux seules dimensions spatiales. Le temporel est présent avec force où l'individuel et le collectif se mélangent subtilement pour créer une ramification incessante de labyrinthes dans un monde chaotique qui mélange réel et imaginaire.

\section{La demeure d'Astérion ${ }^{25}$}

L'originalité de ce labyrinthe est annoncée par le titre même de la nouvelle : le substantif "demeure" évoque plutôt le foyer. Cette désignation est riche de conséquences car le labyrinthe n'est plus une architecture fermée mais une maison dont les portes sont perpétuellement ouvertes. Astérion y vit en toute liberté et y cherche la tranquillité qui fait défaut à l'extérieur. Tout ce qui est autre lui fait peur comme les visages « sans relief ni couleur (88) » des gens au crépuscule. Donc, ce lieu qui lui est propre et qui lui assure de quoi boire et manger, le protège de tout ennui étranger à sa nature. Cette maison « comme il n'en existe aucune autre sur la surface de la terre (87) » partage avec Astérion l'originalité et surtout la monstruosité.

Ce lieu unique en son genre est ouvert « jour et nuit aux hommes et aussi aux bêtes (87) »; les abreuvoirs et les mangeoires l'attestent. En effet, elle est de la même nature double d'Astérion qui est la raison de sa solitude : «Il est exact que je ne sors pas de ma maison. [...]. Ce qu'un homme peut communiquer à d'autres hommes ne m'intéresse pas (87-88)». L'absence d'interaction et d'échange avec les autres humains est en cause. Ceci ouvre le chemin à la notion du labyrinthe et comme le dit Message (2009) : «Si le labyrinthe évoque la solitude, la solitude engendre le labyrinthe ». En fait, la fonction de cet endroit-refuge répond à certaines angoisses de son unique

${ }^{25}$ Borges, La Demeure d'Astérion (L'Aleph, Gallimard, 1967, pour la traduction française). 
habitant. Pourtant, cette demeure labyrinthique est loin de lui assurer la délivrance recherchée. Astérion s'imagine parfois traqué et poursuivi en cherchant vainement quelque chose de vague et d'incertain. La description de ses fréquentes errances le confirme : « Je me cache dans l'ombre d'une citerne ou au détour d'un couloir et j'imagine qu'on me poursuit. Il y a des terrasses d'où je me laisse tomber jusqu'à en rester ensanglanté (89)». Outre les crises d'angoisses qui affligent Astérion, s'ajoute la multiplicité infinie de composantes de ce lieu. Paradoxalement, cette demeure unique souffre de la bifurcation et de la pullulation des formes architecturales qui se répètent interminablement :

Toutes les parties de celle-ci sont répétées plusieurs fois. Chaque endroit est un autre endroit. Il n'y a pas un puits, une cour, un abreuvoir, une mangeoire ; les mangeoires, les abreuvoirs, les cours, les puits sont quatorze [sont en nombre infini] [...] les mers et les temples sont aussi quatorze [sont en nombre infini]. Tout est plusieurs fois, quatorze fois (90).

Donc il est clair que le nombre quatorze symbolise l'infini, dans les deux mondes intérieur et extérieur. La formule divine, composée de quatorze mots, objet de quête pour Tzinacan dans L'Écriture du Dieu l'atteste : accéder à ce chiffre, c'est accéder à l'infini qui est le Tout.

En réalité, cette demeure est à « l'échelle du monde ou plutôt, elle est le monde (89) ». Avec cette expression l'intention de Borges est claire : cette habitation mystérieuse est l'allégorie du labyrinthe existentielle de l'homme dans lequel l'être humain souffre sous le joug de sa double nature, animale et humaine, représentée par Astérion. Cet être exceptionnel et solitaire vit dans son propre monde et ne s'intéresse à aucune interaction avec les autres. Fier de ses origines comme fils d'une reine, il ne peut pas « être confondu avec le vulgaire » [...] [Il est] « unique ; c'est un fait (88)». Bien qu'il regrette son analphabétisme, il ne ressent pas le besoin d'apprendre quelque chose des hommes car « Ce qu'un homme peut communiquer à d'autres hommes ne [1]' intéresse pas [...] [Il est] « comme le philosophe », [il pense] que l'art d'écrire ne peut rien transmettre (88) ». Effectivement, la Bibliothèque de Babel et son savoir chaotique et incommensurable, ainsi que le Livre de sable avec ses pages interchangeables et infinies confirment cette idée : la transmission du savoir par le biais de l'écriture pose une profonde difficulté.

Dans cette solitude effrayante, Astérion imagine un autre Astérion, jouant avec lui, lui parlant de sa maison qui se ramifie à l'infini. Par un tel comportement, il cherche son double selon Lafon (1994) : «Le narrateur monstrueux, tout en proclamant son unicité radicale, joue à se dédoubler (c'est le jeu de l'autre Astérion), il vit dans l'attente de l'arrivée de son double- 
rédempteur $[\ldots]$ il collabore à sa perte ». En fait, la ramification, principe labyrinthique par excellence, touche la personnalité même d'Astérion.

Avec cette coexistence infinie des éléments, «il y a deux choses au monde qui paraissent n'exister qu'une seule fois : là-haut le soleil enchainé ; ici-bas Astérion (90) ». Son originalité, loin d'être un obstacle pour lui, est plutôt une source de fierté confuse. Sa double nature humaine et animale l'enrichit et consolide ses liens avec le cosmos : conscient de sa singularité et de sa force, Astérion s'imagine qu'il est le créateur du soleil et de cette demeure, mais il ne s'en souvient plus. Comme le soleil est source de vie sur la terre, il est devenu la source de délivrance des êtres humains. Sa demeure peut être qualifiée de " labyrinthe de rédemption" car tous les neuf ans, neuf personnes y entrent pour se débarrasser de leurs souffrances. Elles meurent l'une après l'autre dans les couloirs, et leurs cadavres restent comme repères du lieu où chacune d'elles devient une coordonnée dans cet univers bifurqué. C'est là qu'émerge clairement le concept cyclique du temps selon un rythme de neuf ans. Que représente le chiffre 9 pour Borges ? Il est le plus grand chiffre du système de numération de base dix car tout nombre strictement supérieur à 9 sera composé de deux chiffres ou plus. La période temporelle s'impose, puisqu'elle est déterminée non seulement par un cycle de neuf ans, mais aussi par le nombre des êtres humains qui pénètrent volontairement dans cette demeure. Ces entrées régulières et rythmées servent le même objectif. Dans la pensée du narrateur, créateur d'une symbolique particulière des chiffres, cela peut signifier le tout. C'est comme s'il désignait tous les humains qui aspirent à la délivrance et à la paix éternelle. La mort des uns serait un facteur guidant les autres. Le destin de chacun dependrait de ceux de ses dévanciers d'où l'attente de la redemption et ce lien de correlation entre la vie et la mort. Cette dernière devient une action au lieu d'être une soumission à l'anéantissement. Ainsi, l'existence humaine ne peut être exclusivement l'expression de la vitalité présente mais aussi des sacrifices des générations passées.

Par ses valeurs métaphoriques, cette demeure devient un lieu de la renaissance malgré la mort apparente. Les personnes qui entrent trouvent leur délivrance et permettent simultanément à Astérion d'accéder à l'espoir en recevant un message de l'un des neuf êtres humains, qui « au moment de mourir, annonça qu'un jour viendrait [son] rédempteur ». Cet échange au-delà de la vie et de la mort donne un sens bénéfique à cet endroit. Alors Astérion s'investit dans cette idée et commence à imaginer son rédempteur : "Sera-t-il un taureau ou un homme ? Sera-t-il un taureau à tête d'homme ? Ou sera-t-il comme [lui] ? (90)». Cette interrogation aux dimensions philosophiques relie l'Astérion de Borges au Minotaure grec. Pourtant, le labyrinthe du premier diffère complètement du labyrinthe de ce dernier. Borges a changé complètement l'orientation de ce mythe : ni Thésée, ni Ariane n'existent plus. 
Mais surtout, Astérion est fier de sa solitude, il est un peu philosophe, et aucunement prisonnier. Les êtres humains viennent volontairement chez lui et de manière cyclique non comme victimes mais pour se délivrer de leurs supplices en offrant délibérément leur vie. D'ailleurs, les mangeoires et les abreuvoirs sont disponibles en abondance dans cette demeure et le problème de la faim est absent.

Apparemment, les connotations de la demeure d'Astérion diffèrent verticalement, par certains aspects, avec le concept du labyrinthe de base. En effet, s'il s'agissait d'un labyrinthe matérialisé, au sens traditionnel du terme, son architecture serait verrouillée et difficile de s'en affranchir. Quand quelqu'un y accède, il le fait souvent par contrainte et non par consentement d'où son désir de s'en échapper au plus vite afin de survivre. Tandis que ce foyer-labyrinthe est ouvert perpétuellement sur l'extérieur et rien n'empêche d'y entrer ou d'en sortir : les gens y accèdent exprès en cherchant à se délivrer de toutes sortes de malheurs. Astérion, lui-même, qui est libre dans ses allées et venues se comporte en tant que propriétaire des lieux au lieu d'en être le prisonnier. C'est pourquoi le mot labyrinthe n'a pas été mentionné par l'auteur lui-même.

La Demeure d'Astérion fait échos aux concepts de l'immortalité et de la mortalité chez Borges traités dans L'Immortel. En effet, la quête des neufs individus qui entrent chez Astérion serait en rapport avec le concept de l'infini puisqu'ils souffrent du temps interminable et ils s'apaisent dès qu'ils soient dans cette demeure. C'est la mort qui met terme à leurs supplices en abolissant l'infini. Dans les couloirs, les cadavres deviennent des feux de signalisation guidant les vivants sur le bon chemin, c'est-à-dire la mortalité réduit l'aspect labyrinthique de la traversée de ce monde. En effet, une survie interminable fait accroit la misère de l'homme, ce qui le pousse à chercher une issue pour échapper aux dédales de l'infini de la vie humaine, source de stagnation et de répétitions insensées. Cette idée chère à Borges a été étudiée symboliquement et profondément dans la nouvelle L'Immortel qui illustre l'aventure fantastique du Rufus qui après avoir expérimenté l'immortalité, objet de sa quête douloureuse, il rebrousse chemin et retourne vers la condition mortelle source de vitalité et d'humanité.

\section{Conclusion}

Les concepts du labyrinthe chez Borges, qu'ils dépendent du réel ou de l'imaginaire, du monde physique ou du monde psychique, de l'état d'éveil ou du sommeil, du passé ou du futur ont toujours une dimension en commun : l'infini. Ce dernier qu'il soit matériel, spirituel, symbolique, temporel, spatial, destructeur ou salvateur, est intrinsèque à l'idée du labyrinthe.

Tout peut être une matière labyrinthique. Alors, un passé confus, une histoire enchevêtrée, une recherche quelconque, un livre mal écrit, une 
connaissance mal digérée, le monde des savoirs humains, l'ignorance et le fanatisme, les états des peurs, les angoisses et les cauchemars peuvent être des labyrinthes. De la sorte, tout conflit insoluble, toute quête impossible, toute interrogation sans réponses sont des labyrinthes. En effet, la poursuite du savoir par l'homme témoigne de son désir de sortir des labyrinthes de l'inconnu pour saisir les mystères sans fin qui l'entourent. Dans ce sens, l'univers est un labyrinthe, ainsi que tout ce qui est difficile ou impossible à contrôler.

Pourtant, deux types de labyrinthes attirent l'attention par leur particularité : celui du monde terrestre représenté par La demeure d'Astérion et celui de l'univers rêvé par Tzinacan, le prisonnier de L'Écriture du Dieu où « Borges s'égare délicieusement dans son labyrinthe, jusqu'à s'y dissoudre au profit des vérités universelles » selon Mottet (2010). Avec le premier, le labyrinthe peut ne pas être nommé et il n'est pas non plus un lieu d'emprisonnement.

Paradoxalement, s'affranchir d'un labyrinthe n'est pas obligatoirement une sortie pure et simple et sans transformation : souvent le labyrinthe est un processus d'initiation et une situation favorable à la renaissance avant d'être un lieu de souffrance, comme s'il fallait passer par les épreuves du chaos pour aboutir à la création d'un sens. Ainsi, le labyrinthe est loin d'être une perte de temps mais un passage indispensable, une sorte de purgatoire, un microcosme dans lequel l'enfantement d'une idée ou d'un monde est en perpétuel activité. En plus, le labyrinthe en soi n'est pas une calamité absolue. La demeure d'Astérion en est l'exemple : ce labyrinthe original peut représenter partiellement le refuge et la sécurité pour son habitant et même le lieu providentiel pour les chercheurs du salut.

La victoire sur le labyrinthe n'est pas fatalement renier la notion du labyrinthe. Tzinacan s'est libéré de tous ses labyrinthes infernaux pour rejoindre le labyrinthe apaisant, le labyrinthe des labyrinthes qui est l'univers selon Borges. En fait, les différents types de labyrinthes se situent entre le pire mal et la béatitude la plus parfaite, une idée qui fait écho à la notion de l'abîme et du gouffre chez Victor Hugo. La bifurcation et la déviation continuelles sont à l'origine de l'égarement dédaléen qui éloigne sa victime de l'unicité de la vérité radicale et la fait plonger dans la multiplicité répétitive et mensongère. C'est pourquoi, l'épreuve labyrinthique se présente comme une phase initiatique nécessaire à la compréhension de la mortalité qui met terme au recommencement insensé des apparences pour aboutir à l'essence. Cette connaissance progressive rompt la chaîne infernale de l'infini corrupteur pour accéder à l'infini salutaire. Ainsi, le labyrinthe borgésien suit la structure même de l'existence où le destin individuel se fait le miroir de celui de l'humanité toute entière et même de tous les êtres vivants qui participent à l'aventure universelle. 


\section{References:}

1. Borges, J. L. (1944). Fictions. Paris: Gallimard: (Nouvelle Édition Française, 1993).

2. Borges, J. L. (1953). Labyrinthes. Trad. de l'espagnol (Argentine) par Roger Caillois. Paris: Gallimard.

3. Borges, J. L. (1975). Le livre de sable. Traduction revue par JeanPierre Bernès. Paris: Gallimard,Nouvelle édition,1999.

4. Borges, J.-L. (1936(1971)). Historia de la eternidad (Histoire de l'éternité), essai "El tiempo circular" (Le temps circulaire). trad. de l'espagnol par Roger Caillois et Laure Guille, Paris, U.G.E. Paris: UGE.

5. Buttor, M. (1975). Matière de rêve, 1975, Paris, . Paris: Gallimard.

6. Deleuze, G., \& Gattarie, F. (1980). Mill Plateaux. Paris: Édition de Minuit.

7. Duborgel, B. (1982). Espaces en représentation. Centre interdisciplinaire d'études et de recherches sur l'expression contemporaine (Saint-Etienne). Saint-Etienne : Saint-Etienne : CIEREC.

8. Kremer-Marietti, A. (1970). L'homme labyrinthique et la vérité. Les Études Philosophiques(1), 47-61. doi:JSTOR, www.jstor.org/stable/20845690. Accessed 27 Nov. 2020

9. Kremer-Marietti, A. (1999). Nietzsche : l'homme et ses labyrinthes. Paris: L'Harmattan.

10. Lafon, M. (1994). Mythe et fiction: L'Aleph et ses labyrinthes. Bulletin de Littérature Générale et Comparée, 17, 25-42.

11. Lavarde, A. (1999). La sphère, symbole du mouvement. Communication \& Langages(119), 55-65.

12. Martin, J.-C. (2006). Borges : Une biographie de l'éternité (de l'éclat, philosophie imaginaire ed.).

13. Message, V. (2009). Impossible de s'en sortir seul. Fictions labyrinthiques et solitude Chez Franz Kafka, Jorge Luis Borges, Mark Z. Danielewski et t Stanley Kubrick. Amaltea. Revista de mitocrítica, $1,189-201$.

14. Mottet, P. (2010). Les jeux de la réécriture : L'Aleph de Borges ou la re-création du monde. Québec français, 159, 42-45.

15. Mourey, J. (2013). Essais d'Interprétation du conte de Borges « L'Écriture du Dieu ». doi:https://agoras.typepad.fr/regard-éloigné/

16. Nicolas, L. (1999). Borges et l'infini. Variaciones Borges(7), 88-146.

17. Pascal, B. (1954). Pensées. Euvres complètes. Paris: Gallimard.

18. Sager, M. (2014). Qu'est-ce que la philosophie? Affronter le chaos, tracer un plan sur le chaos. Études de lettres(4), 14-24. doi: https://doi.org/10.4000/edl.1100 
19. Sayegh M. (2021). Le chaos borgésien entre réel et virtuel. European Scientific Journal, ESJ, 17 (36), 98-115.

20. Sayegh M. (2021). L'Écriture du Dieu : Libération de la pensée humaine des banalités telluriques. European Scientific Journal, ESJ, 17 (33), 43-65.

21. Sayegh M. (2021). L'Immortel de Borges ou le refus de l'éternité. European Scientific Journal, ESJ, 17(6), 130-149.

22. Taillade, N. (1994). L'œuvre littéraire et le labyrinthe (Le Château de F. Kafka, L'Aleph de J.L.Borges, L'Emploi du temps de M. Butor). In:. Littératures(31), 129-156. doi:: https://doi.org/10.3406/litts.1994.1673

23. https://www.larousse.fr/dictionnaires/francais/labyrinthe/45804\#.

24. https://fr.thefreedictionary.com/dédales.

25. https://blogostelle.blog/category/histoire-du-sacre/

26. Pélissier Augustin, Principes de rhétorique française, http://obvil.parissorbonne.fr/corpus/ecole/pellissier_principes-derhetorique-francaise_1867 https://www.jepense.org/symbolisme-ducercle/ https://agoras.typepad.fr/regard_eloigne/jlborges/ 